\title{
Directional Wettability of Butterfly Wing and Biomimetic Preparation of Superhydrophobic Polymer Film by Soft Lithography
}

\author{
Yan Fang ${ }^{\mathrm{a}}$, Gang Sun ${ }^{\mathrm{b}}$, Dandan $\mathrm{Jin}^{\mathrm{c}}$, Jing Wang ${ }^{\mathrm{d}}$, Yongfang Hou ${ }^{\mathrm{e}}$ and Fei \\ Dong $^{f}$
}

School of Life Science, Changchun Normal University, Changchun 130032, China

afangyan124@aliyun.com, bsungang@nenu.edu.cn, ${ }^{\mathrm{c}} 751599002 @ q q . c o m,{ }^{\mathrm{d}} \mathrm{jjin}$ 66456@163.com, e 2397003691@qq.com, ${ }^{f} 1214507083 @ q q . c o m$

\begin{abstract}
The micro-morphology, superhydrophobicity, adhesive property and chemical composition of the butterfly wing surface were investigated by a scanning electron microscope (SEM), a contact angle (CA) meter and a Fourier transform infrared spectrometer (FT-IR). The wetting mechanism of the butterfly wing was discussed from the perspective of biological coupling. The butterfly wing displays multiple-dimensional structural anisotropism. The micrometric scales constitute the primary structure, the submicrometric vertical ribs and horizontal bridges constitute the secondary structure, and the nano stripes constitute the tertiary structure. The wing surface is of superhydrophobicity (CA 150.4 155.7 ${ }^{\circ}$ ) and low adhesion (sliding angle $1 \sim 4^{\circ}$ ). The water droplet exhibits directional sliding behavior on the wing. The scale plays a crucial role in determining the self-cleaning property of the wing. Using butterfly wing as a bio-template, superhydrophobic polymer (polydimethylsiloxane, PDMS) film was prepared by soft lithography. The rough micro-morphology and wettability of the butterfly wing were replicated very well to the biomimetic film with water CA of $151.5^{\circ}$. The cooperation of hydrophobic material and rough microstructure contributes to the special wettability of the wing. The butterfly wing can be potentially used as a template for biomimetic design of functional surface with complex wettability.
\end{abstract}

Keywords: Wettability, Anisotropism, Preparation, Polymer, Butterfly.

\section{Introduction}

Anisotropism is one of the most important properties of a patterned solid surface. The anisotropic rough surface leads to special wetting and dewetting characteristics in different directions. One example is the anisotropic dewetting phenomenon on the superhydrophobic rice leaf surface. The sliding angle (SA) of a water droplet is greatly influenced by the anisotropic arrangement of the papillae on the rice leaf [1]. The anisotropic wettability has drawn much attention and has been applied for fabrication of self-assembling patterned surfaces, submicrometric channel lattices with alternating wettability and rice-like aligned carbon nanotubes (ACNT) film [2, 3]. The anisotropic dewetting property may bring interesting insights into design of lossless liquid transportation channels and novel microfluidic valves, in which liquids can be driven in a preferred direction. In the present paper we investigated the anisotropism of the rough surface on the butterfly wings and discussed the wetting mechanism from the perspective of biological coupling. This work can not only promote our understanding of anisotropic wetting phenomenon on bio-surfaces, but provide inspiration for design and preparation of smart fluid-controllable interface and directional self-cleaning material.

\section{Materials and Methods}

\subsection{Materials}

The butterfly specimens of ten species were collected in Changchun City, Jilin City and Harbin City of northeast China, and identified by systematic taxonomy. The wings were cleaned, desiccated and flattened, then cut into $5 \mathrm{~mm} \times 5 \mathrm{~mm}$ pieces from the discal cell (Fig. 1). The distilled water for the measurements of contact angle (CA) and SA was purchased from Tianjin Pharmaceuticals Group Co. 
Ltd., China. The volume of water droplet was $5 \mu$ l. The PVA (polyvinyl alcohol) used in the first soft transfer was purchased from Sinopharm Chemical Reagent Co., Ltd, China. The PDMS (Sylgard 184 Silicone Elastomer Kit) used in the second soft transfer was purchased from Dow Corning, USA.

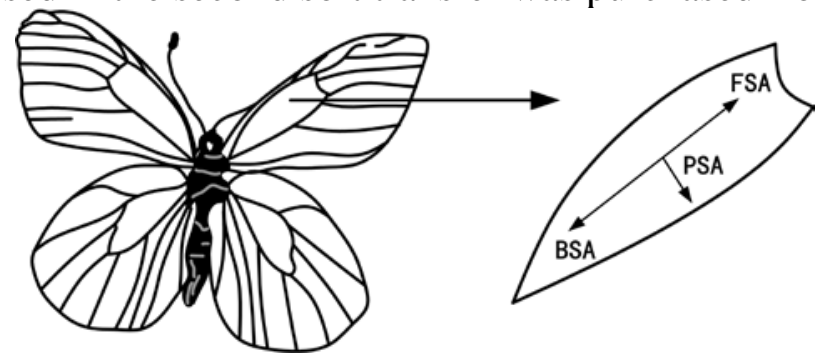

Fig. 1 The experimental area and the SAs of water droplet in different directions FSA: forward SA; BSA: backward SA; PSA: perpendicular SA.

\subsection{Methods}

After gold coating by an ion sputter coater (Hitachi E-1045, Japan), the wing pieces were observed and photographed by a SEM (Hitachi SU8010, Japan). A video-based CA measuring system (DataPhysics OCA20, Germany) was used to measure the CA of water droplet on the wing surface by sessile drop method at room temperature of $25 \pm 1{ }^{\circ} \mathrm{C}$. The SA was measured in three directions, including forward SA (FSA, the SA of droplet from the wing basal to the wing terminal), backward SA (BSA, the SA of droplet from the wing terminal to the wing basal), and perpendicular SA (PSA, the SA of droplet perpendicular to the major axis of the wing) (Fig. 1). The chemical composition of the wing was investigated by means of FT-IR (Nicolet FT-IR200, USA).

In the first soft transfer, the wing piece was affixed to a glass slide with double-sided adhesive tape as the primary template. PVA solution (mass concentration 10\%) was dropped homogeneously on the wing surface. After $24 \mathrm{~h}$ under the ambient temperature of $(25 \pm 1){ }^{\circ} \mathrm{C}$, the PVA film with the inverse structure of the wing surface was peeled carefully off the primary template. In the second soft transfer, the PVA film was affixed to a glass slide with double-sided adhesive tape as the secondary template. The PDMS and curing agent were mixed in a volume ratio of 10:1. The PDMS mixture was dropped homogeneously on the PVA film, then degassed in a vacuum chamber. Having been baked at $120{ }^{\circ} \mathrm{C}$ for $1.5 \mathrm{~h}$, the PDMS was solidified and could be peeled off the secondary template with tweezers.

\section{Results and Discussion}

\subsection{Anisotropism of the Micro-morphology on the Wing Surface}

The wing surface exhibits multiple-dimensional rough structures. The micrometric scales constitute the primary structure. The scale shapes of various butterfly species are similar (Fig. 2A). The length of the scale is $183 \sim 374 \mu \mathrm{m}$, the breadth is $62 \sim 115 \mu \mathrm{m}$, the spacing is $89 \sim 146 \mu \mathrm{m}$. The submicrometric vertical ribs and horizontal bridges on the scales constitute the secondary structure. The vertical ribs and horizontal bridges are linked as grids, some vertical ribs have branches (Fig. 2B). The height of the vertical rib is $527 \sim 912 \mathrm{~nm}$, the breadth is $483 \sim 1026 \mathrm{~nm}$, the spacing is $1628 \sim 2139 \mathrm{~nm}$. The nano stripes distributing regularly on the vertical ribs and horizontal bridges constitute the tertiary structure (Fig. 2C). All the primary, secondary and tertiary structures display remarkable anisotropism.

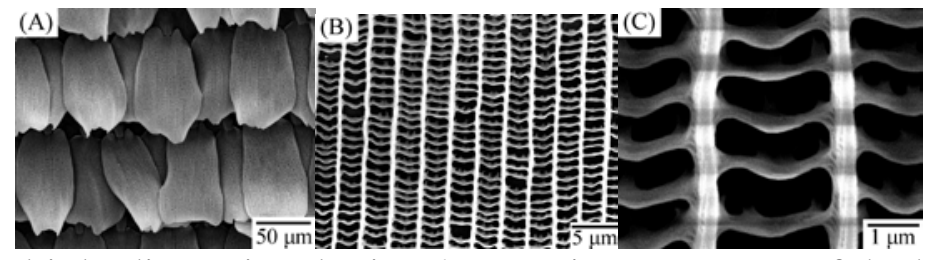

Fig. 2 The multiple-dimensional micro/nano microstructures of the butterfly wing

(A) Primary structure; (B) Secondary structure; (C) Tertiary structure. 


\subsection{Anisotropism of the Self-cleaning Property on the Wing Surface}

The wing surfaces exhibit low adhesion. The range of FSA is $1 \sim 4^{\circ}$, BSA $5 \sim 9^{\circ}$, PSA $4 \sim 7^{\circ}$ (Table 1 ). Meanwhile, the wing surfaces are superhydrophobic $\left(C A>150^{\circ}\right)$. The range of $C A$ is $150.4 \sim 155.7^{\circ}$. The large CA and small SA imply outstanding self-cleaning characteristic of the wing surface. There are extremely significant differences between FSA and BSA $(P<0.01)$, between FSA and PSA $(P<0.01)$, as well as between BSA and PSA $(P<0.01)$. The self-cleaning performance exhibits remarkable anisotropism on the wing surface. The butterfly wing surface is of low adhesive superhydrophobicity.

Table 1 CA and SA of water droplet on the butterfly wing surfaces

\begin{tabular}{|c|c|c|c|c|c|c|c|}
\hline \multirow{3}{*}{ No. } & \multirow{3}{*}{ Butterfly species } & \multicolumn{2}{|c|}{$\mathrm{CA}\left({ }^{\circ}\right)$} & \multicolumn{4}{|c|}{$\mathrm{SA}\left({ }^{\circ}\right)$} \\
\hline & & \multirow{2}{*}{ With scale } & \multirow{2}{*}{ Without scale } & \multicolumn{3}{|c|}{ With scale } & \multirow{2}{*}{$\begin{array}{c}\text { Without scale } \\
\text { FSA/BSA/PSA }\end{array}$} \\
\hline & & & & FSA & BSA & PSA & \\
\hline 1 & Anthocharis cardamines & 151.3 & 112.5 & 2 & 7 & 6 & $>65$ \\
\hline 2 & Aporia hippia & 153.2 & 99.7 & 1 & 6 & 4 & $>65$ \\
\hline 3 & Colias erate & $154.1^{\circ}$ & 112.3 & 4 & 7 & 6 & $>65$ \\
\hline 4 & Euchloe ausonia & 154.4 & 98.5 & 3 & 8 & 5 & $>65$ \\
\hline 5 & Kirinia epaminondas & 150.4 & 114.6 & 1 & 6 & 6 & $>65$ \\
\hline 6 & Leptidea amurensis & 152.8 & 115.4 & 2 & 9 & 4 & $>65$ \\
\hline 7 & Leptidea morsei & 154.3 & 104.7 & 2 & 5 & 5 & $>65$ \\
\hline 8 & Lethe marginalis & 155.2 & 106.9 & 3 & 7 & 6 & $>65$ \\
\hline 9 & Parnassius eversmanni & 151.6 & 105.8 & 2 & 6 & 4 & $>65$ \\
\hline 10 & Pieris melete & 155.7 & 117.2 & 1 & 8 & 7 & $>65$ \\
\hline & Average & 153.2 & 108.8 & 2.1 & 6.9 & 5.3 & $>65$ \\
\hline
\end{tabular}

\subsection{Mechanism of the Anisotropic Wettability on the Wing Surface}

The wing surfaces of different butterfly species have highly similar absorption characteristic of FT-IR spectra. The absorption peaks are at 3287, 2935, 2869, 1643, 1531, 1376, 1223, 1137, 1065 $\mathrm{cm}^{-1}$, respectively. These absorption bands result from stretching vibration, skeletal vibration, deformation vibration or in-plane bending vibration of the bases (e.g. $-\mathrm{CH}_{3},-\mathrm{CH}_{2},-\mathrm{C}-\mathrm{CH}, \mathrm{C}-\mathrm{H}$, $\mathrm{O}-\mathrm{H}, \mathrm{C}=\mathrm{O}, \mathrm{C}-\mathrm{O}, \mathrm{N}-\mathrm{H}$ ) in chitin, protein or fat. The wing surface is composed mainly of naturally hydrophobic material with an intrinsic CA of $95^{\circ}$ [4]. However, much higher hydrophobicity cannot result from the chemical composition alone. Due to the hierarchical rough structures on the wing surface, the water droplet stands on the tips of the vertical ribs. Much air is left under the droplet. The actual contact area between the water droplet and the wing surface is so small that the droplet forms an almost perfect sphere. The solid-liquid-gas triple contact lines (TCL) are expected to be contorted and unstable. The multiple-dimensional microstructure plays a crucial role in the complex wettability of the butterfly wing surfaces. In a contrast test, the scales were removed from the wing surfaces. The CA decreases by 23.8\% 36.2\% (Table 1), all the SAs (FSA, BSA, PSA) increase above $65^{\circ}$ (the maximum inclination angle of the sample table is $65^{\circ}$ ). The low adhesive superhydrophobicity of the wing surface ascribes to the coupling effect of material element and structural element.

\subsection{Micro-morphology and Wetting Property of the Artificial Polymer Film}

The scale on the butterfly (Colias erate) wing is of an oval shape (Fig. 3A). After the first soft transfer, the PVA film with the inverse structure of the wing surface was prepared (Fig. 3B). After the second soft transfer, the PDMS film with the similar structure of the wing surface was prepared (Fig. 3C). The macro/micro structures on the natural wing surface are retained on the PDMS film, resulting in a superhydrophobicity (CA 151.5 $)$. However, the CA of the PDMS film is lower than that of the wing surface $\left(154.1^{\circ}\right)$. This is because only part of the nano structure of the wing surface is replicated on the PDMS film. At present, the copying of nano structure is still one of the difficulties in the field of fabrication of biomimetic materials. 


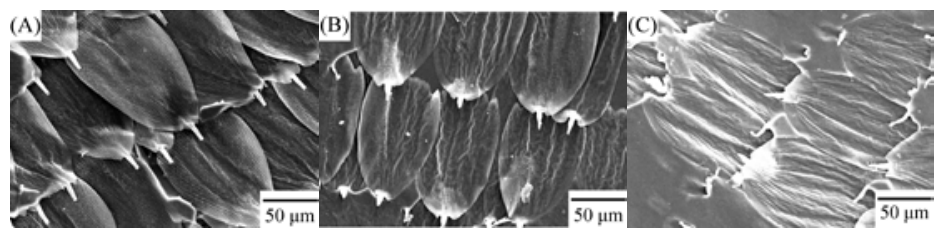

Fig. 3 Micro-morphology of the biomimetic polymer film (A) The butterfly (Colias erate) wing; (B) The PVA film; (C) The PDMS film.

\subsection{Biological Significance of the Anisotropic Property on the Wing Surface}

The anisotropism of SA and self-cleaning property on the wing is the result of the oriented micro-morphology and the energy barrier difference in various directions. When a droplet slides from the wing basal to the wing terminal (FSA), the anisotropic microstructures exert less influence than sliding from the wing terminal to the wing basal (BSA) in hampering droplet sliding, the droplet is highly instable and can roll off more easily, so the SA decreases. This marvelous property results from long-term co-evolution of the butterfly and the environment, and endows the butterfly wings with the ability of directional easy-cleaning in a watery environment. Such a self-cleaning function is of essential biological significance for the butterfly. Even a very slight tilting $\left(1 \sim 4^{\circ}\right)$ is sufficient to cause the water droplet to roll off and take away the contaminating particles from the wing effectively. The butterfly can lighten body burden readily, increase flight stability and efficiency, optimize energy budget. Thus, the butterfly can get more opportunities to survive and thrive.

\section{Conclusions}

The butterfly wing is of low adhesion (SA $1 \sim 4^{\circ}$ ) and superhydrophobicity (CA 150.4 155. $7^{\circ}$ ). The wing is composed of naturally hydrophobic material (chitin, protein, fat, etc.), and possesses hierarchical rough structures. The cooperative effect of material element and structural element leads to the complex wettability of the wing. The butterfly wing exhibits anisotropic micro-morphology and self-cleaning property, which is of critical biological significance for the survival of butterfly. The butterfly wing is an ideal bio-template for development of multi-functional materials. This work not only promotes our understanding of wetting mechanism on bio-surfaces, but brings insights into biomimetic preparation of novel self-cleaning coatings and anisotropic wetting substrate.

\section{Acknowledgments}

This work was financially supported by the National Natural Science Foundation of China (Grant NO. 31671010), the Innovative Program for Postgraduate Students of Changchun Normal University (Grant NO. cscxy2015007, cscxy2017003, cscxy2017006) and the Innovative and Entrepreneurship Program for Undergraduate Students of Jilin Province (201610205079, 201610205014). Dr. Prof. Gang Sun is the corresponding author of this paper.

\section{References}

[1] Y. Fang, G. Sun, T.Q. Wang, Q. Cong, Hydrophobicity mechanism of non-smooth pattern on surface of butterfly wing, Chin. Sci. Bull. 52 (2007) 711-716.

[2] A.M. Higgins, R.A.L. Jones, Anisotropic spinodal dewetting as a route to self-assembly of patterned surfaces, Nature 404 (2000) 476-478.

[3] M. Gleiche, L.F. Chi, H. Fuchs, Nanoscopic channel lattices with controlled anisotropic wetting, Nature 403 (2000) 173-175.

[4] X.J. Wang, Q. Cong, J.J. Zhang, Y.L. Wan, Multivariate coupling mechanism of NOCTUIDAE moth wings’ surface superhydrophobicity, Chin. Sci. Bull. 54 (2009) 569-575. 